\title{
Jahresinhaltsverzeichnis 2018
}

\section{AUFSÄTZE}

B e c k e r, Maximilian: „Keine Waffen für unsere Henker!“ Ehemalige Verfolgte des NS-Regimes und die westdeutsche Wiederbewaffnung . . . . . . . . 87

E d e n, Sören/M a r x, Henry/S c h u l z, Ulrike: Ganz normale Verwaltungen? Methodische Überlegungen zum Verhältnis von Individuum und Organisation am Beispiel des Reichsarbeitsministeriums 1919 bis 1945 . . . . . . . . . 487

E i k e 1, Markus: „Die Herrschaft des Rechts und nicht das Recht des Stärkeren“. Die Bundesrepublik Deutschland und die Entstehungsgeschichte des Internationalen Strafgerichtshofs 1993 bis $1998 \ldots \ldots \ldots \ldots \ldots \ldots \ldots \ldots \ldots \ldots \ldots \ldots \ldots \ldots \ldots \ldots \ldots$

G o l d s t e i n, Yossi: Warum wurde nach dem Sechstagekrieg kein Friede

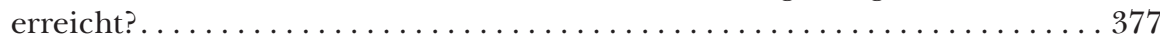

G r a f, Rüdiger: Verhaltenssteuerung jenseits von Markt und Moral. Die umweltpolitische Regulierungsdiskussion in der Bundesrepublik Deutschland und den USA im letzten Drittel des 20. Jahrhunderts . . . . . . . . . . . . 435

H a n n i g, Nicolai: Georg Picht. Strategien eines Medienintellektuellen in der westdeutschen Öffentlichkeit. . . . . . . . . . . . . . . . 617

H o m b e r g, Michael: Mensch | Mikrochip. Die Globalisierung der Arbeitswelten in der Computerindustrie 1960 bis 2000 - Fragen, Perspektiven,

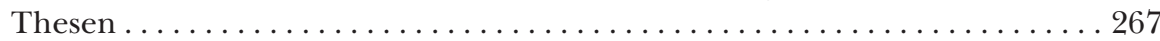

K ö n i g, Malte: „Geschichte ist machbar, Herr Nachbar!“ Die Umbenennung der Berliner Kochstraße in Rudi-Dutschke-Straße 2004 bis 2008 . . . . . . . . . . 463

K o r t ü m, Hans-Henning: „Gut durch die Zeiten gekommen“. Otto Brunner und der Nationalsozialismus . . . . . . . . . . . . . . . . . . . . 117

Ost i G u e r r a z z i, Amedeo: Das System Mussolini. Die Regierungspraxis des Diktators 1922 bis 1943 im Spiegel seiner Audienzen ... . . . . . . . . . . . 201

R e i t e r, Margit: Anton Reinthaller und die Anfänge der Freiheitlichen Partei Österreichs. Der politische Werdegang eines Nationalsozialisten und die „Ehemaligen“ in der Zweiten Republik. . . . . . . . . . . . . . . . 539

R o t h e r, Bernd: Die SPD und El Salvador 1979 bis 1985. Linke Politik im atlantischen Dreieck von Bundesrepublik, Zentralamerika und USA . . . . . . 645 
S c h e r n e r, Jonas: Lernen und Lernversagen. Die „Metallmobilisierung“ im Deutschen Reich 1939 bis 1945 . . . . . . . . . . . . . . . . . . 233

S c h r a f s t e t t e r, Susanna: Zwischen Skylla und Charybdis? Münchner Juden in Italien 1933 bis $1945 \ldots \ldots \ldots \ldots \ldots \ldots \ldots \ldots \ldots 77$

W e i t k a m p, Sebastian: Ein Rückzugsgefecht des Rechtsstaats 1934. Der Prozess gegen SS-Sturmbannführer Heinrich Remmert wegen Häftlingsmisshandlungen im KZ Esterwegen $\ldots \ldots \ldots \ldots \ldots \ldots \ldots \ldots \ldots \ldots \ldots$

W i 11 e m s, Bastiaan: Nachbeben des Totalen Kriegs. Der Rückzug der Wehrmacht durch Ostpreußen und seine Folgen. .

\section{PODIUM ZEITGESCHICHTE}

Wie nah ist uns die Zwischenkriegszeit? Geschichte und Aktualität der demokratischen Staatsgründungen nach dem Ersten Weltkrieg in Deutschland, Österreich, Polen, Litauen und der Tschechoslowakei . . . . . . . . . . . . 295

K o n rá d, Ota: Widersprüchlich und unvollendet. Die Demokratie der Ersten Tschechoslowakischen Republik 1918 bis 1938 . . . . . . . . . . . 337

M a k h o t i n a, Ekaterina: Die Nähe Smetonas. Nationale Identitätskonstruk-

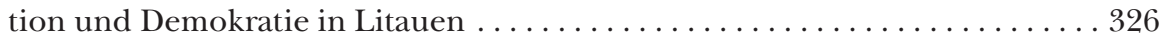

P e 1 i n k a, Anton: Österreich: Fremdbestimmung und Selbstzerstörung. Die lange Suche nach der - nach einer - Identität . . . . . . . . . . . . . 309

R a i t h e 1, Thomas: Noch immer ein Schreckbild? Das heutige Deutschland und die Weimarer Republik . . . . . . . . . . . . . . . . . . . . 299

R u c h n i e w i c z, Krzysztof: Drei Daten, drei Anfänge. Das polnische

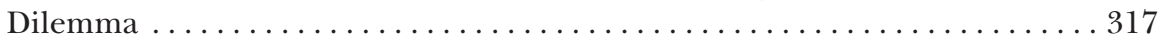

65 JAHRE VfZ

65 Jahre VfZ - Traditionen und Perspektiven $\ldots \ldots \ldots \ldots \ldots \ldots \ldots \ldots \ldots$ 


\section{NOTIZEN}

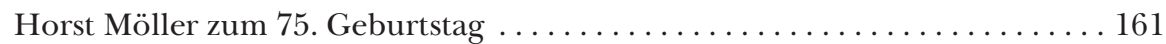

Im Laboratorium der Marktwirtschaft: Zur Geschichte der Treuhandanstalt 1989/90 bis 1994. Ein neues Forschungsprojekt des Instituts für Zeit-

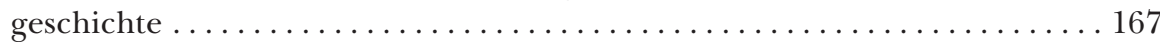

12. Aldersbacher Schreib-Praxis. Ein anwendungsorientiertes Seminar des Instituts für Zeitgeschichte und des Verlags De Gruyter Oldenbourg (23. bis 27. Juli 2018)

Von der Reichsbank zur Bundesbank: Personen, Generationen und Konzepte zwischen Tradition, Kontinuität und Neubeginn 1924 bis 1970. Ein neues Forschungsprojekt des Instituts für Zeitgeschichte in Kooperation mit der London School of Economics and Political Science . . . . . . . . . . . . . . . . . . 349

Wandel der Arbeitswelt - Ökonomische Transformationen, Gewerkschaften und soziale Ungleichheit seit den 1970er Jahren. Ein Graduiertenkolleg als Kooperationsprojekt des Instituts für Zeitgeschichte, des Zentrums für Zeithistorische Forschung und des Instituts für soziale Bewegungen . . . . . . . 685

\section{VfZ-ONLINE}

Dokumentation der Podiumsdiskussion „Wie nah ist uns die Zwischenkriegszeit? Geschichte und Aktualität der demokratischen Staatsgründungen nach dem Ersten Weltkrieg“ ............................... 701 


\section{DE DE GRUYTER \\ OLDENBOURG}

\section{GERMAN YEARBOOK OF CONTEMPORARY HISTORY}

\section{VOLUME 2}

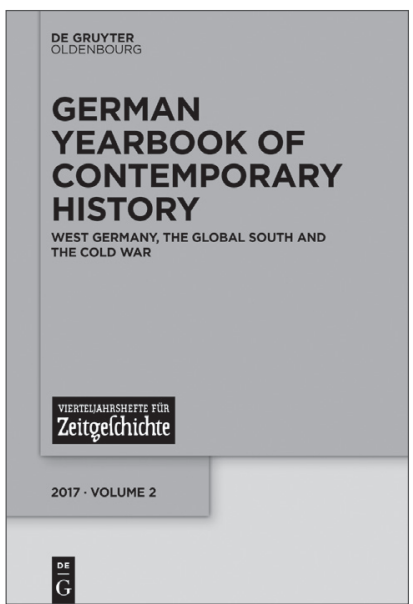

Agnes Bresselau von Bressensdorf, Christian Ostermann, Elke Seefried (Eds.)

WEST GERMANY, THE GLOBAL SOUTH AND THE COLD WAR

2017. 223 pages

HC RRP €49.95 [D]/US \$57.99/£ 45.50

ISBN 978-3-11-052019-4

eBook RRP $€ 49.95[D] /$ US \$57.99/£ 45.50

PDF ISBN 978-3-11-052299-0

ePUB ISBN 978-3-11-052030-9

With its accession to membership of the United Nations in the early 1970s, the Federal Republic of Germany found new scope for its foreign policy, and it was at a time when the global NorthSouth divide became a focus point of international politics. This is the background to the articles in the second volume of the German Yearbook of Contemporary History, edited by two historians from the Institut für Zeitgeschichte (Institute for Contemporary History, Munich-Berlin) - Agnes Bresselau von Bressensdorf and Elke Seefried - together with Christian Ostermann from the Woodrow Wilson International Center for Scholars in Washington. The current yearbook deals with West Germany during a time of Cold War confrontation, issues of human rights and threat from radical Islam. Selected contributions from the quarterly Vierteljahrshefte für Zeitgeschichte offer detailed analyses of West German policies toward Cambodia, Chile, Iran and Afghanistan, and international experts provide a vivid commentary. 